\title{
Frontal Cryosectioning: An Improved Protocol for Sectioning Large Areas of Fibrous Scaffolds
}

\author{
Casey P. Grey ${ }^{1}$ and David G. Simpson ${ }^{2}$ \\ ${ }^{1}$ Department of Biomedical Engineering, Virginia Commonwealth University, Richmond, VA 23284, USA \\ ${ }^{2}$ Department of Anatomy and Neurobiology, Virginia Commonwealth University, Richmond, VA 23298, USA \\ Correspondence should be addressed to Casey P. Grey; greycp@vcu.edu
}

Received 30 January 2015; Accepted 22 April 2015

Academic Editor: Bei Peng

Copyright (c) 2015 C. P. Grey and D. G. Simpson. This is an open access article distributed under the Creative Commons Attribution License, which permits unrestricted use, distribution, and reproduction in any medium, provided the original work is properly cited.

\begin{abstract}
Fibrous tissue engineering scaffolds, such as those produced by electrospinning, cannot achieve their clinical potential until deep cell-scaffold interactions are understood. Even the most advanced imaging techniques are limited to capturing data at depths of $100 \mu \mathrm{m}$ due to light scatter associated with the fibers that compose these scaffolds. Conventional cross-sectional analysis provides information on relatively small volumes of space and frontal sections are difficult to generate. Current understanding of cellular penetration into fibrous scaffolds is limited predominantly to the scaffold surface. Although some information is available from cross-sections, sections vary in quality, can distort spatial scaffold properties, and offer virtually no spatial cues as to what scaffold properties instigate specific cellular responses. Without the definitive ability to understand how cells interact with the architecture of an entire scaffold it is difficult to justify scaffold modifications or in-depth cell penetration analyses until appropriate techniques are developed. To address this limitation we have developed a cryosectioning protocol that makes it possible to obtain serial frontal sections from electrospun scaffolds. Microscopic images assembled into montage images from serial sections were then used to create three-dimensional (3D) models of cellular infiltration throughout the entire scaffold.
\end{abstract}

\section{Introduction}

Electrospinning is a technique that can be used to produce three-dimensional (3D) tissue engineering scaffolds composed of nano- to micron-sized fibers fabricated from natural proteins [1,2], synthetic polymers [3-6], and blends of natural proteins and synthetic polymers [7-9]. The versatility of this technique allows for considerable control over the composition, mechanical (e.g., tensile strength, elasticity, etc.), and structural (e.g., fiber size, fiber alignment, scaffold shape, scaffold porosity, etc.) properties of the resulting constructs $[4,10-12]$. Electrospinning produces a unique class of nanomaterials that can be utilized in a wide variety of biological applications; for example, electrospun scaffolds have been used to investigate how cells interact with $3 \mathrm{D}$ environments of varying compositions [13, 14], and, in a more esoteric application, electrospinning has been proposed as a method for fabricating organs and tissues targeted for replacement therapy [15-17]. Understanding, promoting, and regulating the extent of cellular penetration and population of electrospun scaffolds is fundamental to each of these potential applications and considerable effort has been directed at achieving this objective $[4,8,18]$.

The refractive nature of the fibers that comprise an electrospun scaffold limits the utility of conventional light and confocal microscopy in the analysis of electrospun scaffolds $[19,20]$. Even with two-photon microscopy, the structural properties of these constructs limit imaging to a maximum depth of approximately $100 \mu \mathrm{m}$, and even then the resulting $z$-stack data represents a small fraction of the entire surface area of the scaffold $[20,21]$. Several laboratories, including our own, have used cross-sectional analysis of samples taken from frozen sections in order to judge the extent of cellular penetration into electrospun scaffolds [12, 22, 23]. However, given the complex surfaces and wide distribution of cells in electrospun materials it is difficult to characterize the depth and extent of cell penetration throughout an entire scaffold using these methods. Data collection in this type of analysis 
is usually limited to a few selected sections taken from the scaffold and, without a comprehensive morphometric approach, this type of analysis often results in a subjective and biased analysis of the samples.

Tissue engineering scaffolds targeted for use in reconstructive therapy are typically designed to mimic the gross dimensional characteristics of the native target tissue $[4,10$, $24,25]$. For example, the wall of a native, small diameter artery may reach nearly $500 \mu \mathrm{m}$ in thickness in an adult, ostensibly requiring a scaffold of approximately the same thickness to act as a graft $[26,27]$. In this example, due to the inherent limitations imposed by the structure of the scaffold, the spatial cell-matrix information for the majority of the scaffold would be unobtainable. Developing methods to better understand how cells interact throughout the entirety of a tissue engineering scaffold represents an important element of the design process. Without this type of information it is difficult to make rational decisions concerning what changes in structure, nutrient support, and/or seeding conditions might be necessary to modulate cell distribution and function. Scaffold structural considerations are too often based on limited data (e.g., cross-sectional samples) at best and, at worst, academic guesswork. In this study we describe a method in which we are able to take serial frontal sections through the entire thickness of frozen electrospun scaffolds. This represents a method that provides nearly unambiguous information concerning the depth and spatial distribution of cells within a given scaffold design. By reconstructing data collected from the individual serial sections into a threedimensional model it is also possible to explore how regional variations in scaffold structure impact cell-matrix interactions.

\section{Materials and Methods}

2.1. Electrospinning. All reagents were obtained from Sigma unless otherwise noted. Polycaprolactone (PCL: 65,000 M.W.) was suspended and electrospun from trifluoroethanol (TFE) at concentrations of $150 \mathrm{mg} / \mathrm{mL}$ (producing small diameter fibers) or $250 \mathrm{mg} / \mathrm{mL}$ (producing large diameter fibers). Electrospinning syringes were capped with a blunt-tipped 18gauge needle and placed into a syringe driver (Fisher Scientific) set to deliver the electrospinning fluid into an 18$21 \mathrm{kV}$ static electric field at a rate of $8 \mathrm{~mL} / \mathrm{hr}$ across a $20 \mathrm{~cm}$ gap [4]. A solid cylindrical metal mandrel (length $=11.75 \mathrm{~cm}$, diameter $=6.33 \mathrm{~mm}$ ) or a perforated cylindrical metal mandrel was used as ground targets (functional length $=11.75 \mathrm{~cm}$, mandrel diameter $=6.33 \mathrm{~mm}$, and pore diameter $=0.75 \mathrm{~mm}$ spaced laterally $2 \mathrm{~mm}$ and vertically $1.5 \mathrm{~mm}$ ) [4, 12]. The target mandrels were designed to rotate and translate laterally ( $4 \mathrm{~cm} / \mathrm{s}$ over a $12 \mathrm{~cm}$ distance) in order to facilitate an even distribution of fibers.

2.2. Cell Culture. Human dermal fibroblasts (hDF, Cascade Biologics C-013-5C) were cultured in DMEM-F12 (Gibco) supplemented with $10 \%$ fetal bovine serum (FBS, Hyclone) and $1 \%$ penicillin/streptomycin (P/S, Invitrogen). For cell culture experiments $6 \mathrm{~mm}$ diameter and $1 \mathrm{~mm}$ thick circular samples were punch cut from electrospun scaffolds and sanitized in a $70 \%$ ethanol wash for $30 \mathrm{~min}$ followed by two phosphate buffer solution (PBS) rinses and one media rinse. Scaffolds were seeded with 25,000 cells and cultured in an incubator set to $37^{\circ} \mathrm{C}$ and $5 \% \mathrm{CO}_{2}$. Cell culture media were changed every 3 days. For analysis, populated scaffolds were fixed in $10 \%$ glutaraldehyde in phosphate buffer solution (PBS) for 10 minutes followed by two rinses in PBS. Samples were placed in fresh PBS and stored at $4^{\circ} \mathrm{C}$ until being processed for analysis. Samples were cut into $50 \mu \mathrm{m}$ thick serial sections as described subsequently in this study and stained with $4^{\prime}$,6-diamidino-2-phenylindole (DAPI) and rhodamine phalloidin.

2.3. Image Acquisition. Montage images of each serial section were prepared from data captured with a Nikon TE300 microscope equipped with $10 \mathrm{x}$ and $20 \mathrm{x}$ objectives and a DM 1200 digital camera, all images captured at a resolution of $1280 \times 1024$. Brightfield images were overlaid with fluorescence images using the Photomerge function of Adobe Photoshop CS4. 3D scaffold reconstruction was performed using Google SketchUp Make.

\subsection{Cryosectioning Sample Preparation and Cutting Protocol}

(1) Samples were placed into a $30 \%$ sucrose solution prepared in PBS for 3 days at $4^{\circ} \mathrm{C}$. This extended incubation period was used to ensure that the scaffolds were fully infiltrated with sucrose and cryoprotected during processing. Poor sucrose infiltration (e.g., short soak times) resulted in inconsistent cell preservation and catastrophic sample deformation during cutting (e.g., crush and fragmentation issues that prevented section recovery). This is one of the most critical steps in this protocol; typical procedures call for 1 hour submersion in sucrose which resulted in very poor frontal sectioning [28].

(2) Samples were removed from the sucrose solution and placed directly into a "cryomold" containing sufficient optimal cutting temperature compound (OCT) embedding media to fully submerse the scaffolds. We have found that Fisher, flat-bottom culture dishes work as well as cryomolds. Extended scaffold exposure to unfrozen OCT was avoided as it was found to reduce rhodamine staining of actin.

(3) A nonseeded (scrap) scaffold of identical size to the sample to be processed was placed directly on top of the cell seeded sample. This second scaffold was used as a "buffer" to reduce the risk of physically damaging the underlying scaffold of interest.

(4) The entire cryomold was then placed onto the cryobar inside the cryostat (Figure 1) and, using a press tool, the sample was quickly submerged and flattened by pressing it down to the bottom of the cryomold until all OCT had solidified. In this step the scrap scaffold mitigates physical damage to the scaffold from the press tool and provides a backing for when the press tool is removed (see Figure 2). The press tool is ideally a semirigid, flat-bottom cylinder of similar size to the sample. We used a $5 \mathrm{~mm}$ diameter, $2.5 \mathrm{~cm}$ long cylindrical eraser refill for this study (Helix, Grandville, MI). 


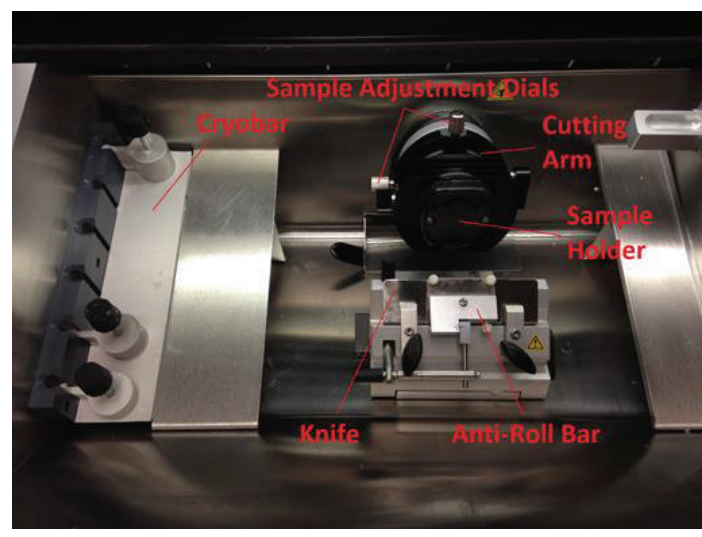

Figure 1: Typical cryosectioning chamber. Temperatures used were as follows: cryobar $\left(-35^{\circ} \mathrm{C}\right)$, chamber $\left(-25^{\circ} \mathrm{C}\right)$, and specimen $\left(-34^{\circ} \mathrm{C}\right.$ to $\left.-36^{\circ} \mathrm{C}\right)$.

(5) Once the OCT in the cryomold had solidified, the press tool was removed with a gentle twisting motion, keeping the sample on the cryobar at all times.

(6) Frozen samples were allowed to fully solidify on the cryobar (3-5 min). At this point all tools needed for sample manipulation (forceps, brushes, knife, etc.) were placed into the cryochamber and allowed to cool to sectioning temperature. Room temperature tools will melt the OCT which can make handling the sample extremely difficult. Additionally any melted OCT that refreezes onto the knife increases cutting difficulty (the knife needs to be as clean as possible at all times).

(7) Frozen scaffolds were recovered from the cryomold and affixed to a textured cryosectioning plate using OCT as an adhesive.

(8) Once the sample was mounted onto the cryosectioning plate, the plate was secured to the sectioning arm of the cryostat and allowed to cool to sectioning temperature (3-5 minutes).

(9) Aligning the sample with the knife edge is crucial. We accomplished this by bringing the sample as close to the knife as possible (without touching) and aligning it as flush as possible to the blade. If the sample is not aligned properly the ensuing cut will yield only a portion of the section. If this happens, realign the sample based on the previous cut and resection. We stored cut sections in 48-well tissue culture plates filled with PBS. Retain any sections that may have been taken misaligned as these and the realigned samples still represent the surface of the scaffold. Useful data can still be extracted from these samples. It takes practice and patience to properly align a scaffold.

(10) For PCL scaffolds, a general sectioning speed of one rotation of the cutting cycle per second was used to process the frozen material. The rate of travel across the knife blade that produces the best sections can be expected to vary to some degree by the nature of the sample processed and is usually determined through experience.
(A) Start with an empty well

(B) Fill the well with OCT

(C) Place the sample in the well (side of interest facing down)

(D) Place the buffer material on top of the sample

(E) Position press tool above the buffer material

(F) Apply enough pressure to flatten the sample

(G) Maintain pressure until the sample is frozen

(H) Remove press tool and allow the sample to freeze completely
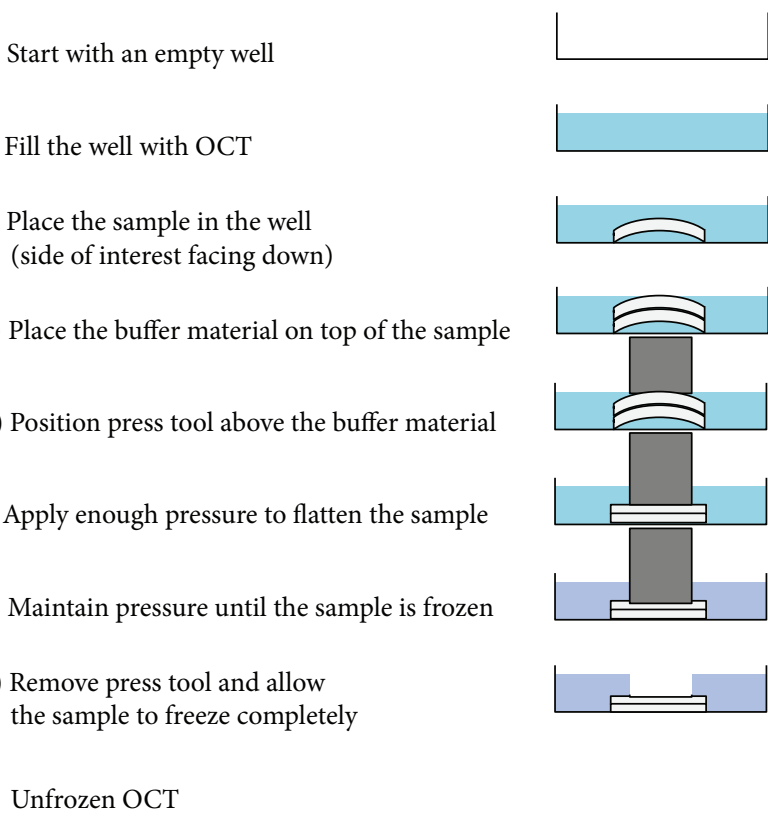

FIGURE 2: Flattening and freezing a sample for cryosectioning. The flattening process is critically important-ensuring the sample is flush with the bottom of the flat bottomed cryomold allows for accurate alignment of the first, crucial section. Steps (A)-(D) are completed outside of the cryosectioning chamber (room temperature) while steps $(\mathrm{E})-(\mathrm{H})$ are completed inside the cryosectioning chamber, on the cryobar or on an equivalent precooled metal mass. Steps (E) and (F) must be completed quickly after placing the sample in the cryosectioning chamber to prevent the premature freezing of the sample in an unflattened state (e.g., if a sample has residual tension, such as when electrospinning onto a small diameter mandrel, it will tend to curl).

(11) Once cut, each section was recovered from the knife surface with cold, fine-tipped forceps by grasping the frozen OCT that surrounds the cut section (rather than grasping the section itself) so as not to damage it. Each section was then placed in separate PBS-filled wells (24-, 36-, and 48-well plates work well for this process), rather than onto dry microscope slides. This serves a dual purpose; first, placing sections directly into PBS quickly dissolves any residual OCT and ensures that the sections stay hydrated; the hydration step improves overall image quality. Second, dissolving the residual OCT mitigates the concerns regarding reduced rhodamine staining mentioned above. Third, the PBS bath is very forgiving and helps to flatten or unfurl sections that may have folded during recovery. Manually flattening a section after placement onto a dry microscope slide is extremely difficult as the delicate sections tend to tear rather than unfold. Additionally, rehydration of cut sections allowed to dry on a microscope slide commonly results in air bubbles being trapped inside the fibrous network, reducing image quality. 


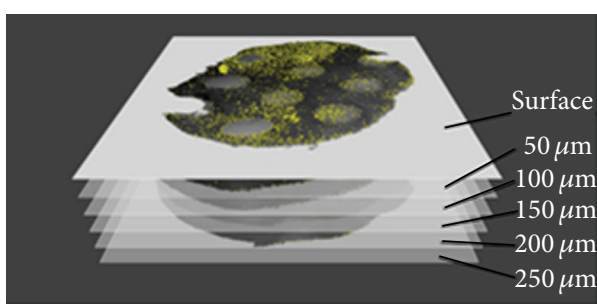

(a)

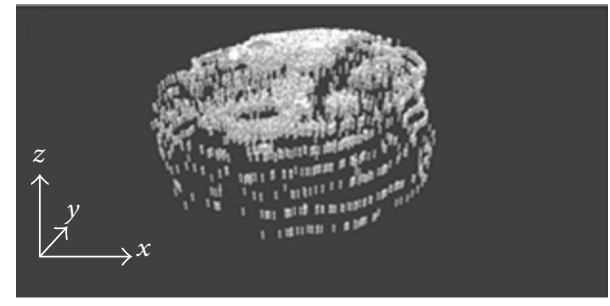

(c)

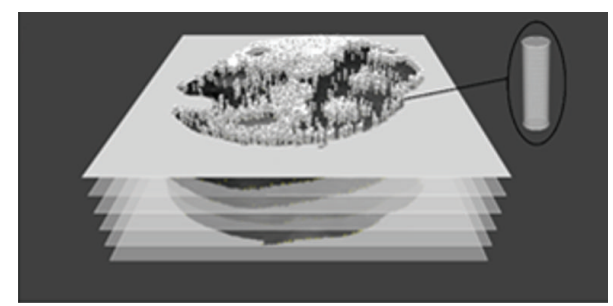

(b)

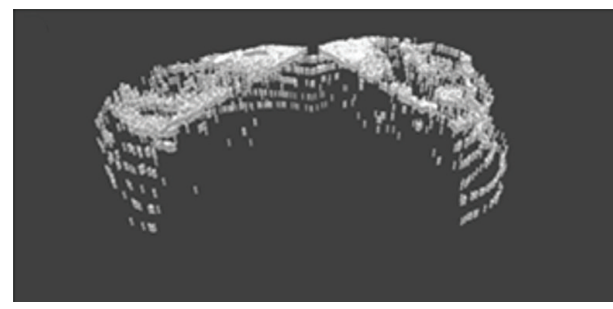

(d)

Figure 3: Construction of a three-dimensional model of cellular infiltration. (a) Images are imported into the workspace and separated on the $z$-axis by a factor of 10. (b) A three-dimensional object of choice is used to replace each of the DAPI positive nuclei to represent cell location in space. (c) The bright field channel images depicting the scaffold fibers are turned off. (d) To better visualize cell penetration within the scaffolds a digital section was prepared and the opposing images were rotated to reveal the distribution of cells within the scaffold.

(12) Sections were either stored in PBS at $4^{\circ} \mathrm{C}$ (assuming slow scaffold degradation rates) or processed immediately. Regardless of treatment, all cut sections were washed with fresh PBS to remove residual OCT (which can reduce rhodamine staining as mentioned above). To image sections, a drop of PBS was placed onto a microscope slide or coverslip into which (using fine-tipped forceps) sections were transferred. Some less-stiff sections will fold in on themselves once they are moved from the wells but generally unfold once resubmerged in the PBS droplet, manual manipulation was rarely required with the PCL scaffolds. A coverslip was placed over the section and any excess PBS was removed so that the sections were flattened. The sections were then imaged and either placed back into its original well for storage or the coverslip was sealed and the section stored on the slide. Of note, placing the sections between two microscope coverslips (eliminating the thicker microscope slide) for imaging simplifies flipping the sections over to investigate either side of the scaffold in detail.

2.5. Image Analysis. Our objective is to develop methods to characterize the distribution of cells within an electrospun scaffold on a global scale. To achieve this objective we systematically captured overlapping images across the entire surface of each individual $50 \mu \mathrm{m}$ thick cryosection. Prior to moving the image field of view images were first captured in the brightfield channel and then in the fluorescence channel; this insures registry between the two image data sets. Paired bright field and fluorescence images were combined to produce a composite image. Using the Photomerge function in Photoshop the individual composite images were then assembled into a montage that represented the entire surface area of each individual serial frontal section. The brightfield channel was used in this assembly process because images collected from the fluorescence channel (especially if DAPI images are required) lack sufficient data density to for the Photomerge function to assemble the montage (i.e., an image of widely distributed nuclei does not have enough image terrain features to allow the algorithm to assemble the individual microscopic image fields into a montage).

Montage images from each individual frontal section were imported into Google SketchUp and placed in a $z$-stack orientation within the workspace (Figure 3). To facilitate modeling we increased the distance between each frontal section by a factor of ten to better visualize scaffold layer properties. Montage composite images were aligned in the vertical orientation using scaffold features or fiduciary marks placed within the scaffolds prior to embedding. This process was greatly simplified in this study because the exemplar scaffold was fabricated using air impedance electrospinning, a process that produces large scale features that penetrate the scaffold in the $z$-direction. Registry marks can be incorporated into the peripheral edges of conventional scaffolds using a small diameter needle to facilitate the alignment process.

To model cell position a 3D shaded rendering of a cylinder approximating the cross-sectional dimensions of a cell was created and used to replace the DAPI nuclear images marking the position of each cell within the scaffold (Figure 3(b)). We note here that the 3D shape used for cellular representation in this model is arbitrary. In some cases it may be helpful to use different shapes, such as spheres, to improve cell visualization; however, it should be noted that this is only a representation of the location of the cell, not a depiction of actual cell shape.

Once all of the cells have been represented by a shaded cylinder (or other 3D object) in the montage $z$-stacks, the brightfield image channel was turned off (Figure 3(c)) allowing just the 3D distribution of the cylinders that mark each 


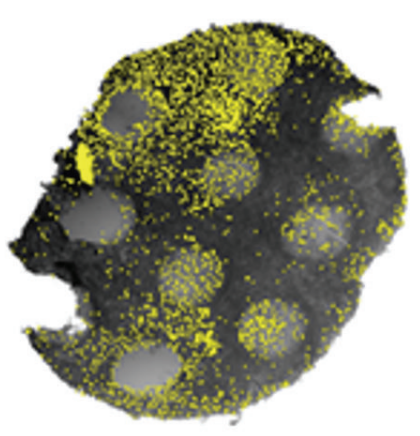

Section depth

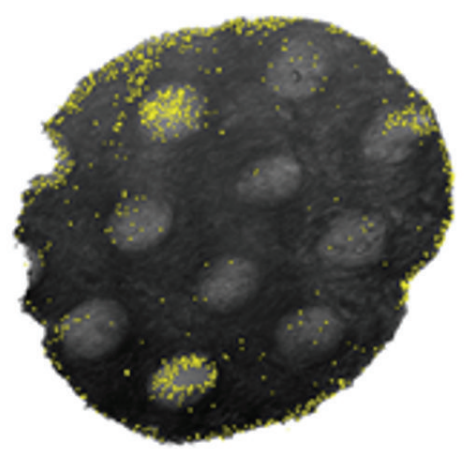

$35 \mu \mathrm{m}$

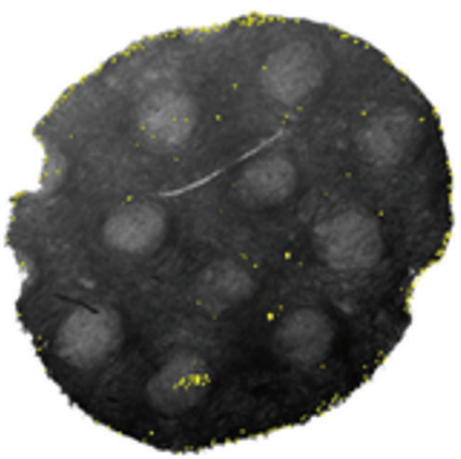

$70 \mu \mathrm{m}$

FIGURE 4: Consecutive frontal images of electrospun scaffolds seeded with human dermal fibroblasts stained with DAPI (yellow). Note how data management can be monitored by examining sections prior to image acquisition. Once some predetermined threshold of cells has been detected in the scaffold frontal sections the data sets are prepared for $z$-stack assembly. In this example, the majority of cells were present in the first $70 \mu \mathrm{m}$ of the sample and subsequent frontal sections were excluded from the analysis. The large scale pores present in this scaffold were used to align the samples in the vertical direction prior to $z$-stack assembly.

cells' position to be visible. Because cells tend to primarily occupy the surface and sides of the represented scaffold we created a digital slice in the scaffold and rotated each half away to better reveal the position of cells that were embedded within the depth of the electrospun scaffold (Figure 3(d)).

\section{Discussion}

3.1. Inadequacies of Current Techniques. Conventional confocal optical imaging techniques are limited to approximately the first $100 \mu \mathrm{m}$ of a fibrous scaffold and, as a result, cannot adequately provide data on the distribution of cells on a global scale within a large construct. Conventional crosssectional analysis (sections taken perpendicular to the surface of a scaffold) can theoretically obtain data similar in nature to the data generated by a frontal section technique. While this is possible, pragmatically frontal sectioning has several advantages compared to conventional cross-sectioning. First, sections taken with this modified cryosectioning protocol retain better structural integrity and are subject to less physical damage. Second, only relevant frontal sections need be analyzed. As a hypothetical example, cells are seeded onto the top surface of an electrospun sample that is $6 \mathrm{~mm}$ in diameter and $1 \mathrm{~mm}$ thick. Then, assume this particular scaffold supports the infiltration of cells to a depth of $200 \mu \mathrm{m}$ (cell penetration to this depth is common in our lab when seeding onto scaffolds consisting of large fibers-data not shown). It would require taking 120 total $50 \mu \mathrm{m}$-thick conventional cross-sections to capture data from the entire scaffold as cells would be present in each cross-section. From a practical perspective, it is unlikely that all 120 sections of this scaffold would be recovered, leaving gaps in the data set. In contrast, if frontal sections were taken, stained with DAPI, and assayed, the fluorescence marking the presence of cells would be present in only the first 5-6 frontal sections (Figure 4). Capturing data from only the relevant sections of the sample simplifies data acquisition and accelerates image processing time. To summarize this example, crosssectioning the entire scaffold would require 120 total sections, all of which would have to be stained and imaged whereas frontal sectioning would require 20 total sections, only 5 of which would have to be stained and imaged.

Storing frontal sections is also more convenient compared to storing conventional cross-sections. In the example described and using 48-well plates to store sections (each section in one well) from each scaffold, cross-sectioning would require 3 complete plates to store one scaffold whereas, using frontal sectioning, 2 separate scaffolds could be stored in a single plate. Because fewer wells are used, the total amount of imaging reagent (e.g., DAPI or other fluorescence markers) required for staining the frontal sections is greatly reduced compared to the conventional sections (despite cross-sectioned samples having a smaller volume, the amount of reagent necessary to cover a sample is dependent on the sample thickness which, in this case, is the same). From a data output standpoint, cross-sections depict the extent of cellular infiltration at a specific location (as does confocal microscopic analysis); data obtained from a frontal section reflects how local features impact the depth of penetration over a large surface area. This type of data might otherwise be lost in a conventional cross-section. In our study, despite the large scale nature of the pores induced by air impedance electrospinning that were present in the scaffolds, it was not possible to clearly identify these features in conventional cross-sectional analysis whereas they are easily distinguished in frontal sections (Figure 5).

3.2. 3D Modeling. As noted, cell distribution and the extent of cell infiltration can be rapidly determined from individual frontal sections (Figure 4). In this figure the scaffold sections have been aligned, placed side-by-side for examination, and projected with the appropriate depth and scaling 


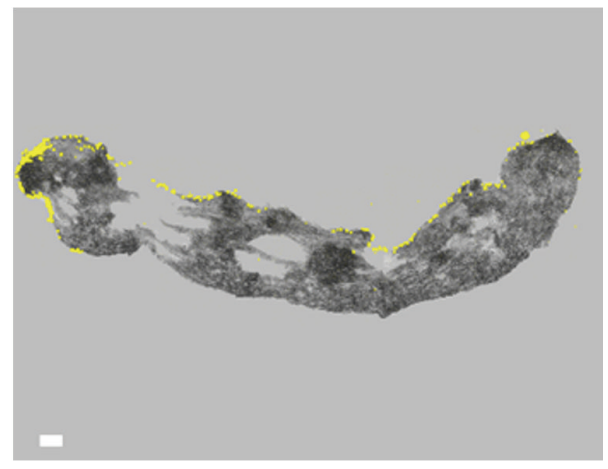

(a)

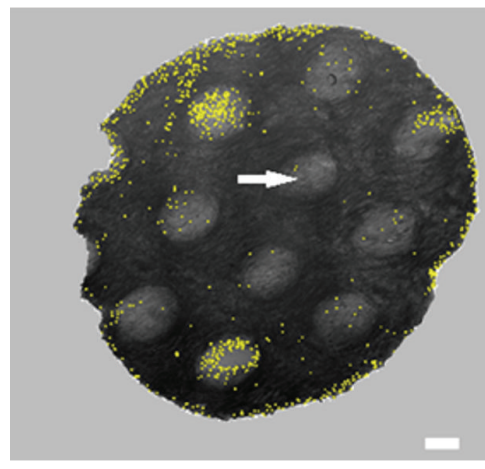

(b)

Figure 5: Cross-section (a) and frontal section (b) of an air impedance scaffold seeded with human dermal fibroblasts (yellow). Notice how the spatial scaffold properties, such as the macropores (arrow), are easily visible in the frontal section whereas in the cross-section no landmarks can be confidently identified. The ability to recognize scaffold features (e.g., macropores) improves our ability to correlate certain cellular events (e.g., infiltration) with changes in scaffold architectures. Left scale bar $=200 \mu \mathrm{m}$ (for (a), cross-section image), right scale bar $=500 \mu \mathrm{m}$ (for (b), frontal section image).

legends. However, frontal sections are highly amenable to $3 \mathrm{D}$ reconstruction and modeling. Entire scaffolds may be reconstructed in this manner.

Insufficient interfiber spacing and low scaffold porosity (despite the enormous void space that is present in these constructs) are commonly cited as factors that limit the penetration of cells into an electrospun scaffold. With caveats, our limited observations in this study largely support these assumptions. Scaffolds produced by air impedance electrospinning exhibit regions of lower fiber density and increased interfiber distances (Figure 4, $35 \mu \mathrm{m}$ deep frontal section) which support localized areas of increased cellular penetration. However, this same analysis reveals that scattered cells are present in regions adjacent to these large scale features. It is unclear at this time if these scattered cells represent cells that have directly penetrated the scaffold or if these cells represent a population that first entered the large scale pores and then migrated laterally to reach these domains.

\section{Conclusions}

We described a modified cryosectioning protocol that addresses a major limitation in the analysis of electrospun scaffolds. This method provides unambiguous data concerning the extent to which cells penetrate a scaffold on a global scale. Through the utilization of this frontal cryosectioning protocol researchers will be able to make definitive conclusions regarding how cellular infiltration and ECM interactions are influenced by specific scaffold structural properties. This information will lead to better scaffold design and an improved understanding of cellular infiltration.

\section{Conflict of Interests}

The authors declare that there is no conflict of interests regarding the publication of this paper.

\section{Acknowledgment}

The authors would like to thank the entire VCU microscopy staff for their assistance with this work.

\section{References}

[1] J. A. Matthews, G. E. Wnek, D. G. Simpson, and G. L. Bowlin, "Electrospinning of collagen nanofibers," Biomacromolecules, vol. 3, no. 2, pp. 232-238, 2002.

[2] D. G. Simpson, B. S. Jha, C. E. Ayres et al., "Electrospun collagen: a tissue engineering scaffold with unique functional properties in a wide variety of applications," Journal of Nanomaterials, vol. 2011, Article ID 348268, 15 pages, 2011.

[3] P. A. Madurantakam, C. P. Cost, D. G. Simpson, and G. L. Bowlin, "Science of nanofibrous scaffold fabrication: strategies for next generation tissue-engineering scaffolds," Nanomedicine, vol. 4, no. 2, pp. 193-206, 2009.

[4] C. P. Grey, S. T. Newton, G. L. Bowlin, T. W. Haas, and D. G. Simpson, "Gradient fiber electrospinning of layered scaffolds using controlled transitions in fiber diameter," Biomaterials, vol. 34, no. 21, pp. 4993-5006, 2013.

[5] M. J. McClure, S. A. Sell, D. G. Simpson, B. H. Walpoth, and G. L. Bowlin, "Tri-layered electrospinning to mimic native arterial architecture using polycaprolactone, elastin, and collagen: a preliminary study," Journal of visualized experiments, vol. 47, article 2084, 2011.

[6] M. A. Woodruff and D. W. Hutmacher, "The return of a forgotten polymer-polycaprolactone in the 21st century," Progress in Polymer Science, vol. 35, no. 10, pp. 1217-1256, 2010.

[7] C. B. Weinberg and E. Bell, "A blood vessel model constructed from collagen and cultured vascular cells," Science, vol. 231, no. 4736, pp. 397-400, 1986.

[8] I. Woods and T. C. Flanagan, "Electrospinning of biomimetic scaffolds for tissue-engineered vascular grafts: threading the path," Expert Review of Cardiovascular Therapy, vol. 12, no. 7, pp. 815-832, 2014. 
[9] Y. Zhang, H. Ouyang, T. L. Chwee, S. Ramakrishna, and Z.-M. Huang, "Electrospinning of gelatin fibers and gelatin/PCL composite fibrous scaffolds," Journal of Biomedical Materials Research. Part B Applied Biomaterials, vol. 72, no. 1, pp. 156-165, 2005.

[10] C. P. Barnes, S. A. Sell, E. D. Boland, D. G. Simpson, and G. L. Bowlin, "Nanofiber technology: designing the next generation of tissue engineering scaffolds," Advanced Drug Delivery Reviews, vol. 59, no. 14, pp. 1413-1433, 2007.

[11] E. D. Boland, B. D. Coleman, C. P. Barnes, D. G. Simpson, G. E. Wnek, and G. L. Bowlin, "Electrospinning polydioxanone for biomedical applications," Acta Biomaterialia, vol. 1, no. 1, pp. 115-123, 2005.

[12] M. J. McClure, P. S. Wolfe, D. G. Simpson, S. A. Sell, and G. L. Bowlin, "The use of air-flow impedance to control fiber deposition patterns during electrospinning," Biomaterials, vol. 33, no. 3, pp. 771-779, 2012.

[13] L. Moroni, R. Licht, J. de Boer, J. R. de Wijn, and C. A. van Blitterswijk, "Fiber diameter and texture of electrospun PEOT/PBT scaffolds influence human mesenchymal stem cell proliferation and morphology, and the release of incorporated compounds," Biomaterials, vol. 27, no. 28, pp. 4911-4922, 2006.

[14] S. H. Lim and H.-Q. Mao, "Electrospun scaffolds for stem cell engineering," Advanced Drug Delivery Reviews, vol. 61, no. 12, pp. 1084-1096, 2009.

[15] U. Boudriot, R. Dersch, A. Greiner, and J. H. Wendorff, "Electrospinning approaches toward scaffold engineering-a brief overview," Artificial Organs, vol. 30, no. 10, pp. 785-792, 2006.

[16] J. Lannutti, D. Reneker, T. Ma, D. Tomasko, and D. Farson, "Electrospinning for tissue engineering scaffolds," Materials Science \& Engineering C, vol. 27, no. 3, pp. 504-509, 2007.

[17] M. Horst, S. Madduri, V. Milleret, T. Sulser, R. Gobet, and D. Eberli, "A bilayered hybrid microfibrous PLGA-Acellular matrix scaffold for hollow organ tissue engineering," Biomaterials, vol. 34, no. 5, pp. 1537-1545, 2013.

[18] B. M. Baker, A. O. Gee, R. B. Metter et al., "The potential to improve cell infiltration in composite fiber-aligned electrospun scaffolds by the selective removal of sacrificial fibers," Biomaterials, vol. 29, no. 15, pp. 2348-2358, 2008.

[19] F. Pampaloni, E. G. Reynaud, and E. H. K. Stelzer, "The third dimension bridges the gap between cell culture and live tissue," Nature Reviews Molecular Cell Biology, vol. 8, no. 10, pp. 839845, 2007.

[20] D. Semwogerere and E. R. Weeks, "Confocal microscopy," in Encyclopedia of Biomaterials and Biomedical Engineering, Taylor \& Francis, 2005.

[21] B. M. Whited, M. C. Hofmann, P. Lu et al., "Dynamic, nondestructive imaging of a bioengineered vascular graft endothelium," PLoS ONE, vol. 8, no. 4, Article ID e61275, 2013.

[22] P. Deshpande, R. McKean, K. A. Blackwood et al., "Using poly(lactide-co-glycolide) electrospun scaffolds to deliver cultured epithelial cells to the cornea," Regenerative Medicine, vol. 5, no. 3, pp. 395-401, 2010.

[23] H. M. Powell and S. T. Boyce, "Engineered human skin fabricated using electrospun collagen-PCL blends: morphogenesis and mechanical properties," Tissue Engineering Part A, vol. 15, no. 8, pp. 2177-2187, 2009.

[24] C. E. Ayres, B. S. Jha, S. A. Sell, G. L. Bowlin, and D. G. Simpson, "Nanotechnology in the design of soft tissue scaffolds: innovations in structure and function," Wiley Interdisciplinary Reviews: Nanomedicine and Nanobiotechnology, vol. 2, no. 1, pp. 20-34, 2010.
[25] B. S. Jha, R. J. Colello, J. R. Bowman et al., "Two pole air gap electrospinning: Fabrication of highly aligned, three-dimensional scaffolds for nerve reconstruction," Acta Biomaterialia, vol. 7, no. 1, pp. 203-215, 2011.

[26] S. B. Kritchevsky, T. Shimakawa, G. S. Tell et al., "Dietary antioxidants and carotid artery wall thickness: the ARIC study," Circulation, vol. 92, no. 8, pp. 2142-2150, 1995.

[27] C. Xu, R. Inai, M. Kotaki, and S. Ramakrishna, "Electrospun nanofiber fabrication as synthetic extracellular matrix and its potential for vascular tissue engineering," Tissue Engineering, vol. 10, no. 7-8, pp. 1160-1168, 2004.

[28] D. Brown, J. Lydon, M. McLaughlin, A. Stuart-Tilley, R. Tyszkowski, and S. Alper, "Antigen retrieval in cryostat tissue sections and cultured cells by treatment with sodium dodecyl sulfate (SDS)," Histochemistry and Cell Biology, vol. 105, no. 4, pp. 261-267, 1996. 

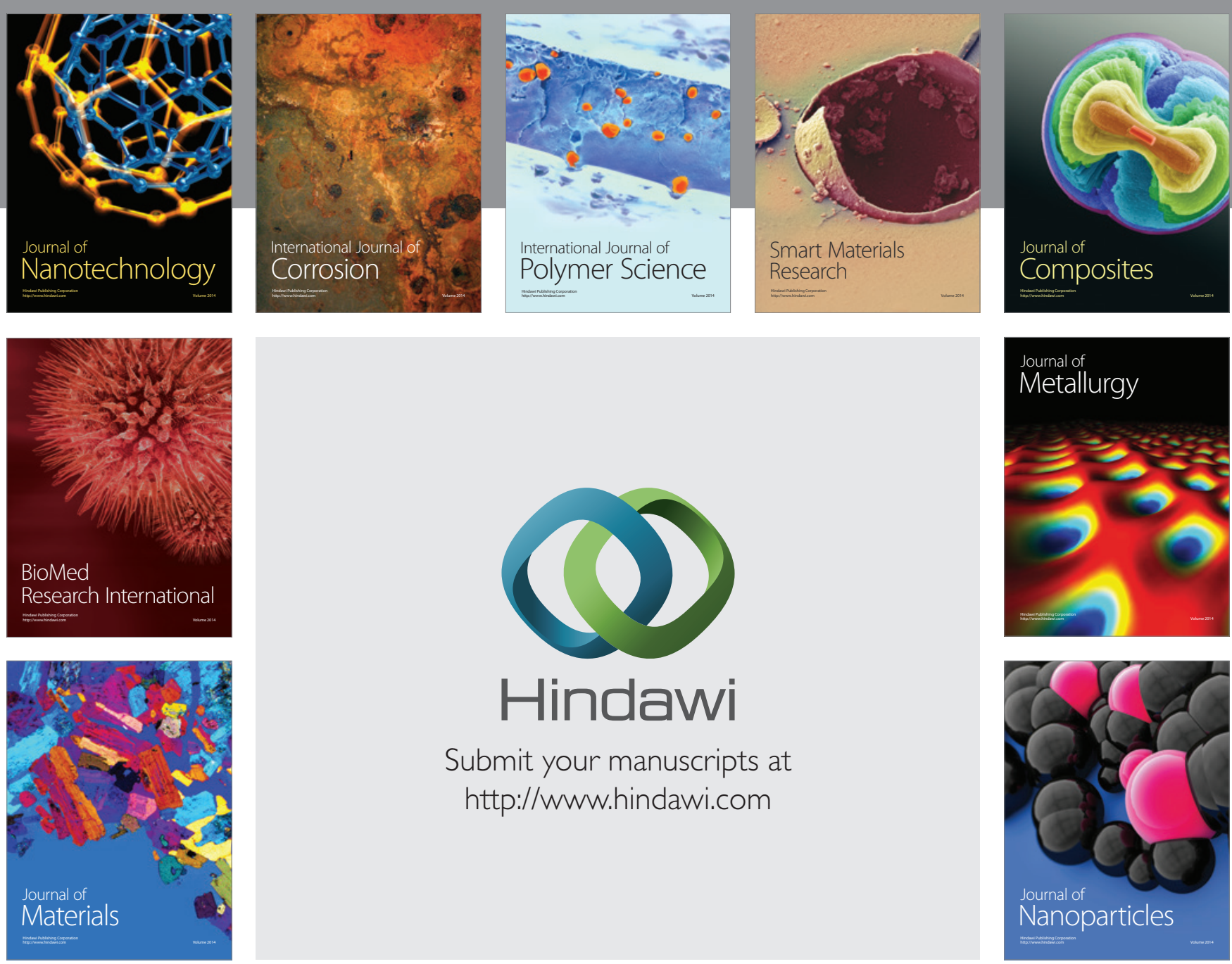

Submit your manuscripts at http://www.hindawi.com
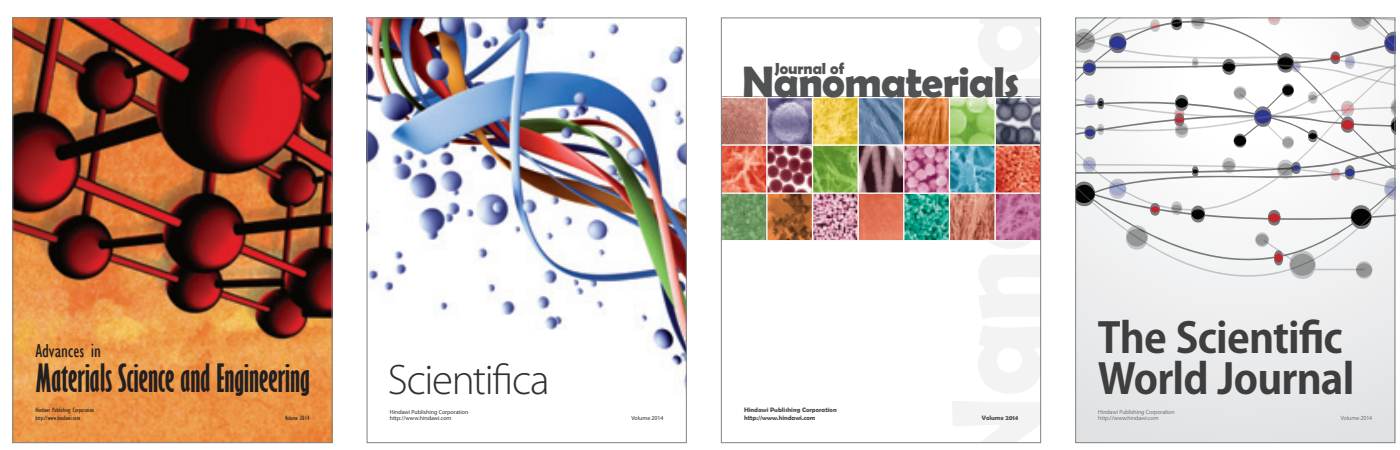

\section{The Scientific World Journal}
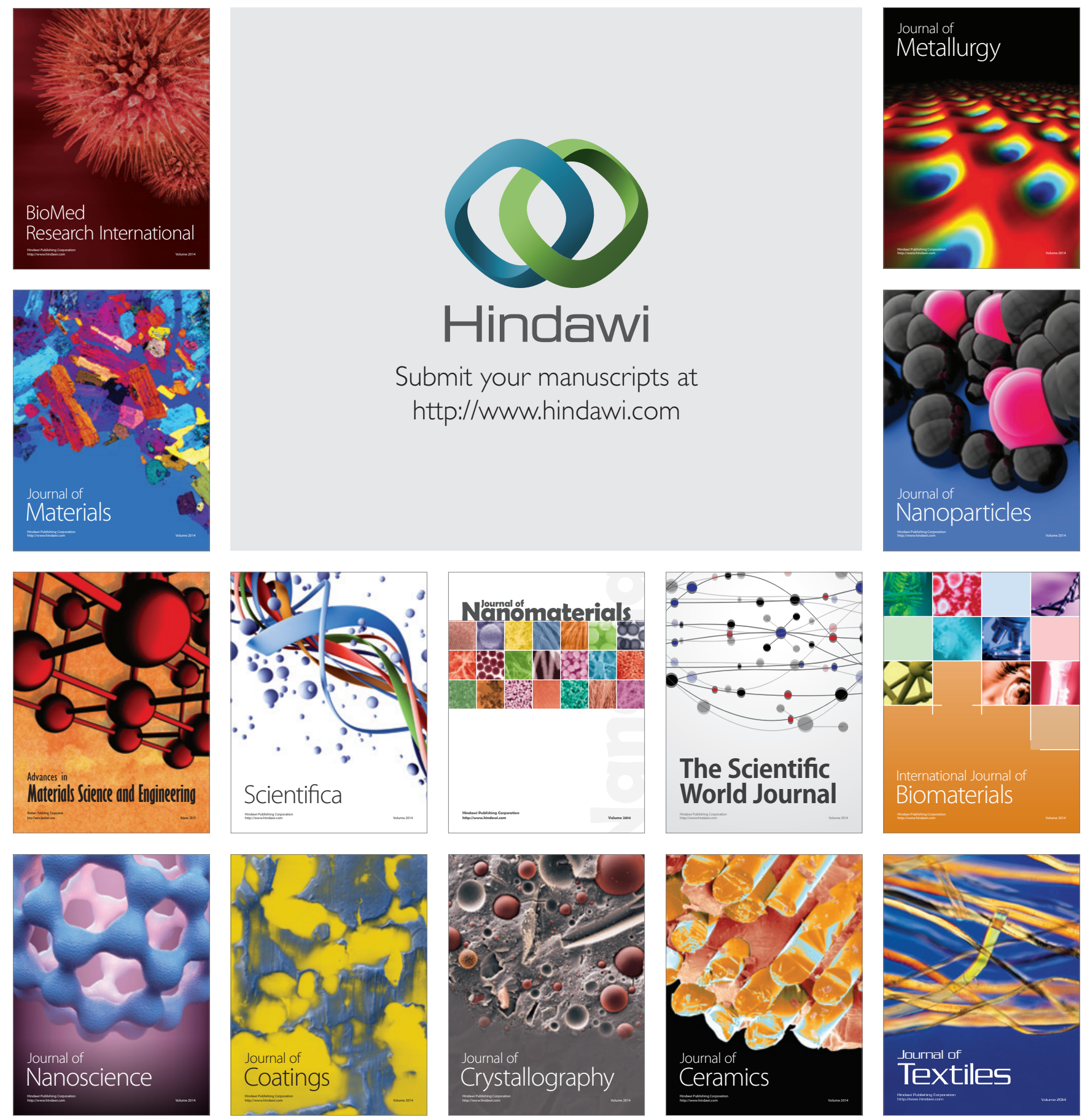